\title{
Buffering Strategies in Optical Packet Switching
}

\author{
Chitra Sharma, Akhilesh Kumar Yadav \\ Research Scholar, UPTU Uttar Pradesh, India \\ E-mail: chitrasharma100192@gmail.com,Akhillakh78@gmail.com
}

\begin{abstract}
Optical packet switching is one of promising technology for the next generation high speed data transfer. In OPS contention among the packets is a major problem, to counteract the problem deflection and buffering of contending packets is proposed. In this paper, a six node network is considered and usability of both deflection routing and buffering of packets is discussed. The results are obtained through simulations, in terms of packet loss probability and average delay. This has been found that in general buffering of contending packets is better option in comparison to deflection. However, in case of load balancing, deflection routing is desirable as some packets take alternative routes to reach its destination. Load balancing scheme reduces the packet loss rate at contending node but it increases the total delay.
\end{abstract}

Index Terms-OPS, Packet loss rate, buffering, deflection routing.

\section{INTRODUCTION}

OPS is a transmission technology which utilizes the enormous bandwidth of optical fiber very efficiently using WDM technology. Thus OPS is considered as next generation data transfer technology. However, the due to the technological limitations it took a while before optical components came into existence.

The invention of LASER gave birth to first generation of light wave communication system. Development of fibers operating at $1.55 \mu \mathrm{m}$ and $10 \mathrm{~Gb} / \mathrm{s}$ with attenuation of $0.2 \mathrm{~dB} / \mathrm{km}$ and due to evolution of optical components implementation of fast growing optical networks is possible. Deployment of Dense Wavelength Division Multiplexing (DWDM) is possible due to introduction of optical amplifiers (e.g. Erbium Doped Fiber Amplifiers, EDFA). Optical transmission and switching technologies based on wavelength division multiplexing (WDM) is extensively used in the Internet infrastructure over the last few years in order to meet the increasing demand for bandwidth.

As bandwidth keeps increasing, electronic switches which perform the switching functionality electronically, convert the optical input signal to the electronic domain, and then convert this signal back to the optical domain, will become complicated and expensive. Hence, the speed of electronics will not be able to keep up with the fast growing bandwidth availability. These problems were driving the migration to implement switching functionality in the optical domain. Key component in fast optical switches is the switching matrix, where optical signals are routed from a certain input port to the destination output port. Over the entire path in packet switched network the payload is retained in the optical domain from source to destination. Several architectures of these switching matrices are possible [1-6]. Some of these have the drawback of being internally blocking, limiting the throughput, and thus increasing the packet loss in such a node.

Packet switches based on photonics have some potential advantages over their electronic counterparts. Studies have shown that the ultimate capacity of photonics-based switches will exceed the capacity of large electronic switching nodes [3]. Another advantage offered by photonics is optical transparency which means that, except for the packet header, the packet payload can be encoded in an arbitrary format and at an arbitrary bit rate. In addition, to increase the switching performance Wavelength Division Multiplexing (WDM) can be exploited since more than one packet can be carried by different wavelengths at the same time and the same input/output port.

Major difficulty in the implementation of optical packet switching systems is the lack of optical Random Access Memory (RAM). Due to unavailability of optical RAM the alternative is to use optical fiber delay lines with other optical components such as optical gate switches, optical couplers and amplifiers to realize optical packet buffering [4-6]. Several different technologies have been developed for optical packet switching. Designing of these architectures is based on parameters like way of optical buffering, the placement of optical buffers, the way of solving the external blocking and the components used to implement the WDM.

In optical networks edge routers acts as a interface between client and core network. These edge routers have $\mathrm{E} / \mathrm{O}$ and $\mathrm{O} / \mathrm{E}$ conversion capability. The core network is optical in nature. In core network, when two or more than two packets try to occupy the same output link, then contention happens. To resolve contention, either deflection routing or buffer of contending packets is proposed.

The goal of this paper is to analyze the effect buffer on the packet loss probability and average delay. Monte Carlo simulations indicate the performance over wide range of load to visualize the complete picture. Simulation is performed for both shared and output buffering scheme. Results are also obtained with or without load balancing scheme. 


\section{RELATED WORK}

For the buffering of contending packets, various switch designs are proposed with various buffering schemes. Some of the them are detailed below:

R. Srivastava et.al. (2007), proposes a switch where, multiple packets are stored using fiber loop. Here for buffering output buffering scheme is considered.

R. Srivastava et.al. (2008), proposes a switch design where shared buffering is efficiently used for the buffering of contending packets. The major problem of the design is huge splitting loss, thus re-circulation count becomes a major issue.

Jun Huang et.al., (2011), even in this design multiple packets are stored, but switch design is very complex.

R. Srivastava et.al. (2010), proposes a switch based on AWG, this design is very efficient, with very low switch insertion loss.

Houman Rastegarfar, et.al., (2013) proposes a switch based on AWG, this design is modified version of design presented in [5]. Here packets are stored in shared manner and can be made re-circulate in the buffer. However, in this design WDM is lost in the buffer.

\section{BUFFERING SCHEMES}

Packet switching is a network communications method that groups all transmitted data into suitably-sized blocks, called packets. The data packets are of fixed size and some header bytes are added before transmission. Header contains information such as destination address, sender address, size of payload etc. The data packets of message are transmitted by one node to another till it reaches its destination. As the header has to be processed at each switch node, it is desirable that header has a relatively low fixed bit rates. The use of fixed length packets can significantly simplify the packet contention resolution and buffering, packet routing as well as packet synchronization. The packet switching does not require a link with a dedicated and reserved bandwidth. Depending on the network and on the available bandwidth on different line any packet may take several possible routes to reach the destination. In such a case, a sequence number is included in the packets to ensure that the packets will not be misinterpreted if they arrive out of order.

Contention among the packets arises when two or more than two packets try to occupy same outgoing fiber. Buffering is a phenomenon, by which contending packets are stored in fiber delay lines. Various fiber delay lines designs are possible where contending packets are placed [5-13]. These designs are well known as optical switches. In these switches buffering can be done at the input, output or within the switch. Input buffering scheme leads to the head on blocking, thus not preferred [14-15]. In OPS two most preferred buffering schemes are output and shared buffering schemes.

In general optical fiber delay lines based buffers can be classified into two categories: travelling-type and recirculating type.

- A travelling-type buffer generally consist of multiple optical fiber delay-lines whose lengths are equivalent to multiples of a packet duration $T$, and optical switches to select delay lines.

- The re-circulating type buffer is more flexible than the travelling type buffer because the storage time of packet can be adjusted by changing the number of revolutions of the data in the buffer. In principle recirculating type buffer acts like random access memory where storage time depends on the number of circulations.

\section{A. Output Buffering}

In output buffering scheme (Fig. 1), a separate buffer is placed at the each output port of the switch and arriving packets are transferred to appropriate output port and will be processed such that FIFO can be maintained. In the output queuing structure no internal blocking occurs. Therefore large throughput is achievable [7].

\section{Output Buffering Scheme}

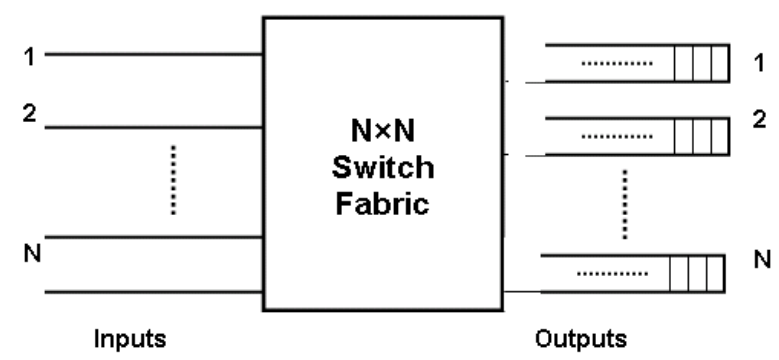

Fig.1. Schematic of Output Buffering Scheme

Output buffering gives the best achievable waiting time performance compared to any other queuing approach. However, for this approach also the large buffer size requirement is a matter of concern, because the larger the required buffer size, the larger would be the cost.

Shared Buffering Scheme

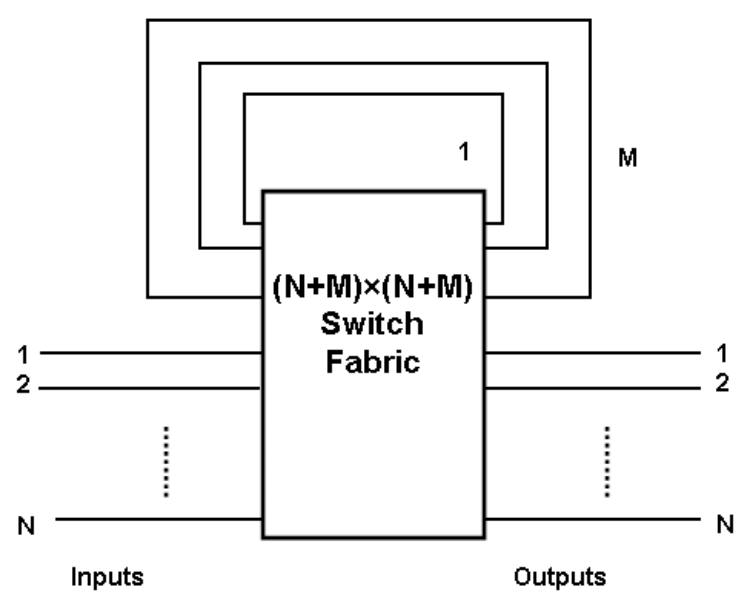

Fig.2. Schematic of Shared Buffering Scheme 


\section{B. $\quad$ Shared Buffering}

The above discussed output buffering schemes are not cost efficient because at each port separate buffer is required. Therefore hardware complexity is very large. In the shared buffering scheme (Fig. 2) packets for all the outputs are stored in the common shared buffer. Therefore hardware complexity is less and still higher throughput is achievable.

Here, in effect, a separate queue is formed for each output of the switch, but physically, all queued packets in the switch share the same buffer space. Due to this kind of queuing, we get advantages of output buffer queuing i.e., less waiting time and at the same time saving a lot of buffer space. However, packet loss probability of switch using output buffer queuing is a little better than shared buffer queuing, but that is immaterial with respect to the amount of buffer space saved due to the shared buffer technique.

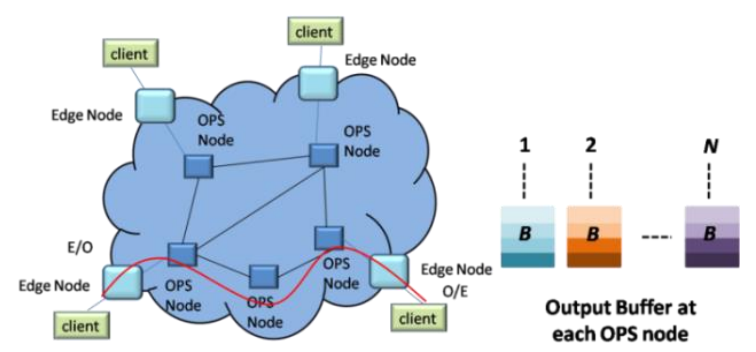

Fig.3. Schematic of OPS Network with Output Buffer at Each Node

In this work we will only consider shared and output buffering of the contending information. Schematic of OPS network with output buffer at each node is shown in Fig. 3. Here, at each node with ' $N$ ' input and output links a maximum of ' $B N$ ' contending packets and for each output ' $B$ ' packets can be stored separately. In figure 4, schematic of OPS network with shared buffer at each node is shown. Here, at each node with ' $N$ ' input and output links can store a maximum of ' $B$ ' contending packets can be stored in shared manner.

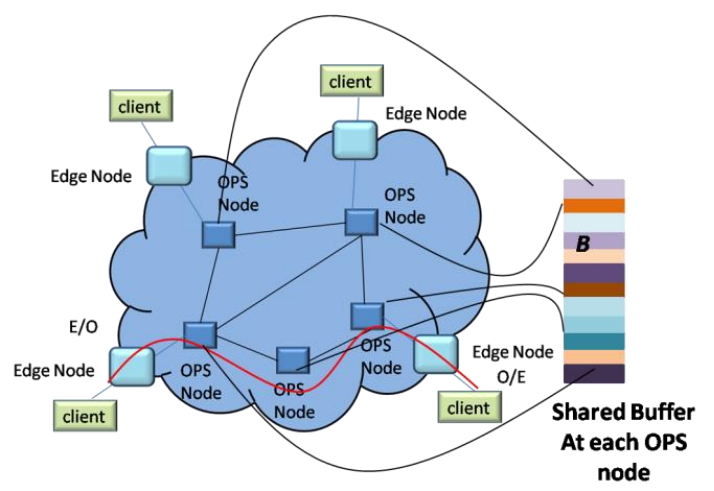

Fig.4. Schematic of OPS Network with Shared Buffer at Each Node

\section{Load Balancing}

Using the above discussion, the optical packet switch will be placed on the nodes R1-R6 and thus buffering will be implemented on these nodes only.
As shown in the figure 5, there are more than one route to reach node $\mathrm{D}$ form node $\mathrm{C}$. In networks distance is counted in terms of number of node traversed and known as hops. So from $\mathrm{C}$ to $\mathrm{D}$ the shortest path is R1-R3-R4. The other two paths R1-R2-R3-R4 and R1-R2-R5-R4 four hops away. As from $C$ to $D$ the shortest path is $R 1-$ R3-R4, most of the traffic will follow this path and sooner or later this path will become congested and either packet will be buffered or they will be dropped. To solve congestion some packet my take longer route to reach its destination and thus path R1-R3-R4 is becomes free after sometime, this phenomenon is known as load balancing.

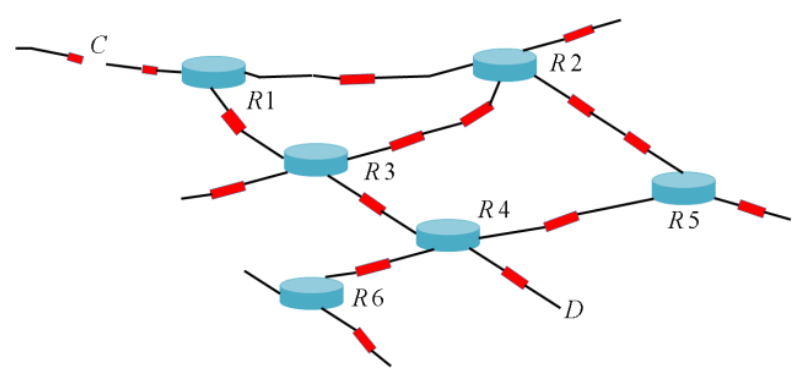

Fig.5. Schematic of OPS Network with Load Balancing Scheme

\section{Simulation Methodology}

This section presents the simulation methodology. The important network parameters are:

Network Throughput: It refers to the volume of data that can flow through a network, or in other words, the fraction of the generated request which can be served.

Network Load: In networking, load refers to the amount of data (traffic) being carried by the network.

Network Delay: It is an important design and performance characteristic of data network. The delay of a network specifies how long it takes for a request to travel across the network from one node or endpoint to another. Delay may differ slightly, depending on the location of the specific pair of communicating nodes.

\section{A. Simulation Framework}

The simulation is performed in MATLAB. The simulator is a random event generator and well known as Monte Carlo simulation. In the simulation random traffic model is considered. In the simulation we assumed the following

1. Request can be generated at any of the input with probability $p$.

2. Each request is equally likely to go to any of the output with probability $\frac{1}{N}$, where $N$ is the number of outputs.

The probability that $K$ requests arrive for a particular output is given by 


$$
P[K]={ }^{N} C_{K}\left(\frac{p}{N}\right)^{K}\left(1-\frac{p}{N}\right)^{N-K}
$$

In the simulation synchronous network is considered hence time is divided into slots. Therefore following assumptions are made.

1. The requests can be generated at the slot boundary only.

The simulation steps are shown in figure 6 to 8 .

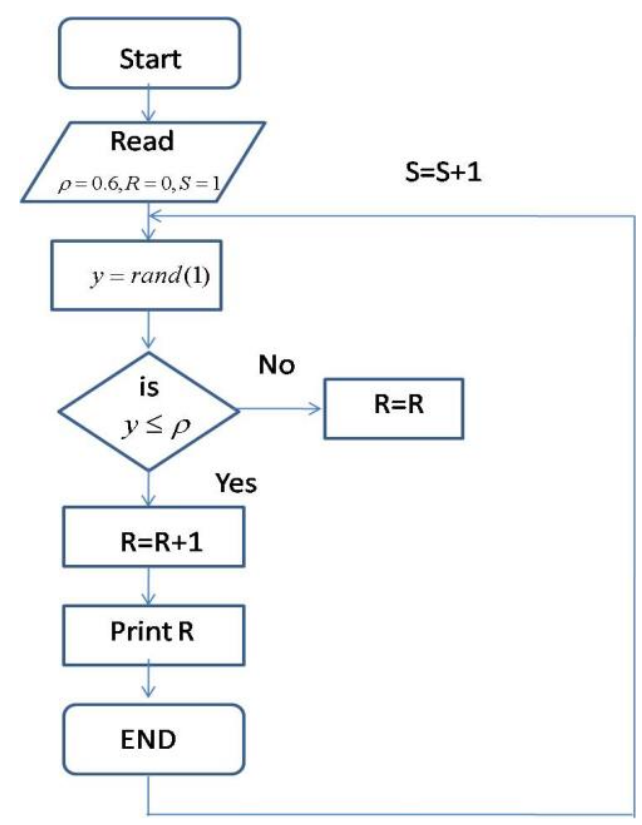

Fig.6. Flow Chart for Request Generation

In fig. 6 flowchart for generation of information is described. The parameters are as defined below:

- $\mathrm{R}=$ Number of request generated (packets)

- $\rho=$ load

- $\mathrm{S}=$ Slot for Simulation

- $\mathrm{Y}=$ Random number

The figure, 6 shows the flow chart for the request generation. Here, for a particular slot a random number ' $y$ ' generated and compared with the considered load (for example 0.6 in figure) if condition is true than a request is generated, otherwise it is considered that no new request is generated. This process is again repeated for the next slot, until the simulation completes.

In figure 7 the flow chart for the destination assignment is shown. Over here is a request is generated then a destination is assigned randomly. In the model uniform random structure is considered.

In flow chart 8 , the buffer management process is described. Over here, first buffer is checked and if there is already pending request in the buffer, then it will be served over incoming request. If buffer is free, i.e., no pending request in the buffer, then it will be served. It is noticeable that the buffered request will be given priority over incoming requests. If request cannot be placed in the buffer, the arriving requests are drooped.
- $\mathrm{R}=$ Number of request generated (packets)

- $\rho=$ load

- $\mathrm{S}=$ Slot for Simulation

- $\mathrm{Y}=$ Random number

- $\mathrm{J}=$ Assigned Destination

- $L=$ Loss

- $B=$ Size of buffer

- $C=$ Counter of packets in the buffer

- $D=$ Delay of packets

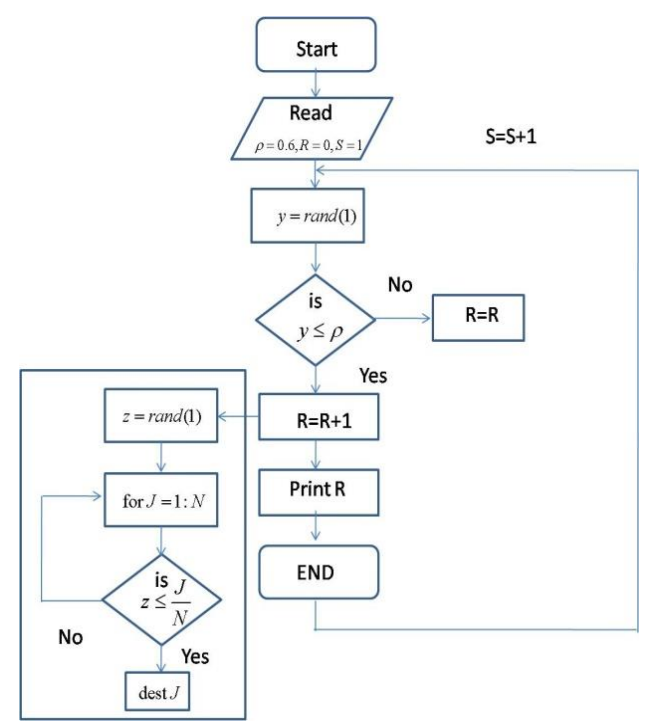

Fig.7. Flow Chart for Request Generation and Server Assignment

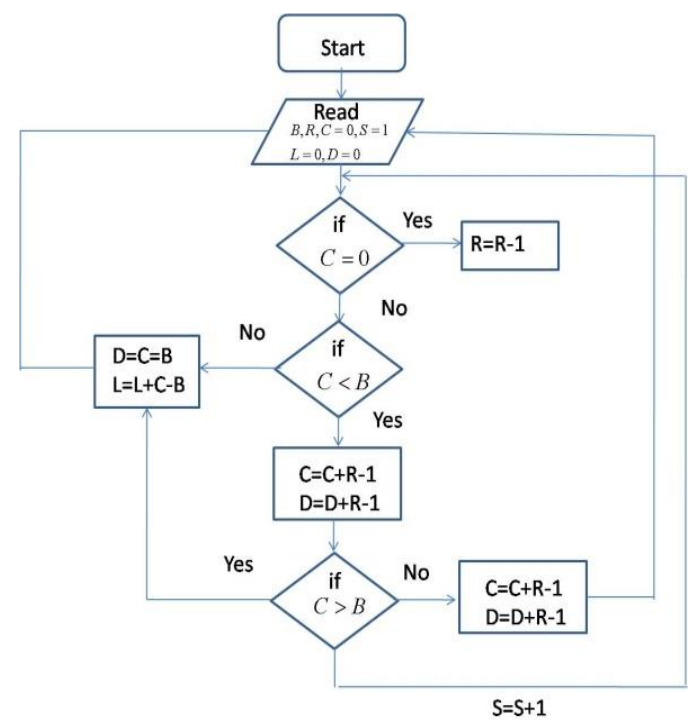

Fig.8. Flow Chart for Buffer Assignment

\section{RESULTS}

In the simulation we have considered a 6 nodes bidirectional network. Here the source node is node 1 while destination node is node 6 .

The shortest route is 1-5-4-6-2, with travelled distance as 1.36. In general it is assumed that the distance between the adjacent nodes will be some hundreds of kilometers. Therefore the travelled distance would be $1.36 \times 1000=1360 \mathrm{~km}$. 
Assuming data propagation speed in fiber as $2 \times 10^{8} \mathrm{~m} / \mathrm{s}$. Then the propagation time would be $(1360 \times 1000) / 2 \times 10^{8}=6.8 \mathrm{~ms}$.

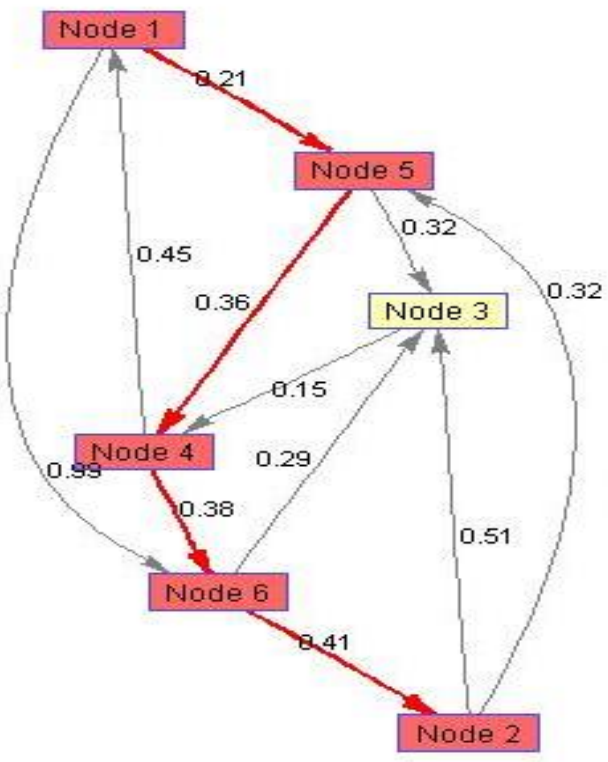

Fig.9. Network under Consideration

Considering that in core network, packet is of size 100000 bits and generation rate is $10 \mathrm{Gbps}$. Then unit slot duration is $100000 / 10^{10}=10$ micro second. Considering the buffering of 16 packets, then the maximum buffering time would be 160 micro second.

Therefore, network propagation time much larger in comparison to buffering time.

Considering, a node in the network with 4 inputs and outputs lines without any buffer. Here, at the load of 0.8 , 25 to 30 percent packets lost, depending on the size of the switch or number of input links (Fig.10).

This loss is huge, and to save these packets either deflection routing or buffering can be used. In deflection routing no extra hardware is required, while in case of buffering some mechanism is needed at each node to buffer the contending packets.

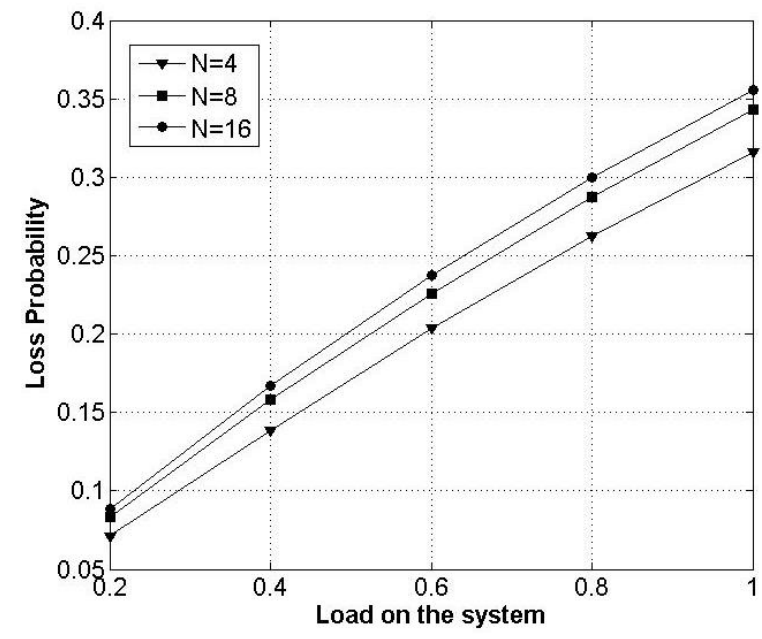

Fig.10. Four Inputs and Outputs Node Without and Buffer
Considering, network in Fig.9, let from node 5 and node 3 two packets arrive for node 4 , then one packet will be processed and other will be deflected. Let packet from node 3 is processed then packet from node 5 will be deflected towards the node1 from where it will be either send back to node 4 or will again be deflected towards node 5. Thus looping may occur, packet will reach at the same node where it was generated after traversing a distance of $1020 \mathrm{~km}$. therefore deflection routing is not a good idea in optical networks.

The buffering of contending packets is done in either shared manner or in dedicated buffering scheme. This dedicated buffer can be placed either at the input or at the output of the switch. The input buffering scheme is not preferred due to the head on blocking. Therefore in this work, output and shared buffering scheme is considered.

\section{A. Output Buffering:}

In Fig. 11-14, simulation results in terms of packet loss probability and average delay for output buffering is discussed.

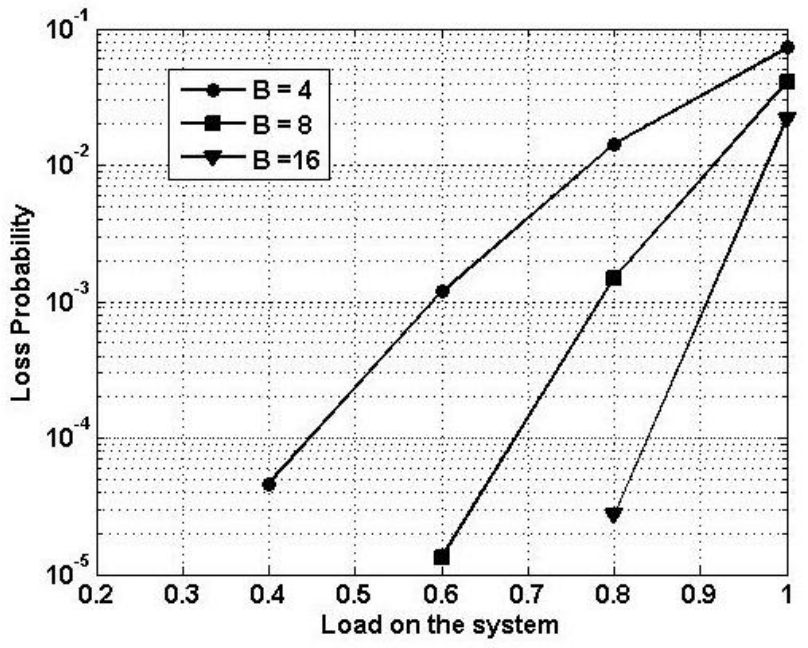

Fig.11. Packet Loss Probability vs. Load on the System with Changing Buffering Capacity

In figure 11, packet loss probability vs. load on the system is presented while assuming $4 \times 4$ nodes with buffering capacity of 4,8 and 16 packets for each node. It is observable that as the load increases the packet loss probability also increases. However, as the buffering capacity increases the packet loss probability decreases. At the moderate load of 0.6 , with buffering of 16,8 and 4 packets, the packet loss probability is approximately, $3 \times 10^{-5}, 2 \times 10^{-3}$ and $1.5 \times 10^{-2}$ respectively. Therefore it can be summarized that the buffering capacity has deep impact on the over-all packet loss probability.

In Fig. 12, average delay vs. load on the system is while assuming $4 \times 4$ nodes with buffering capacity of 4,8 and 16 packets for each node. It is obvious from the figure as the load increases the average delay increases. It can also be visualized form the figure that as the buffering capacity increases the average delay also increases. At lower and moderate load $(<0.6)$ average 
delay is independent of buffering capacity. As load crosses 0.8 mark, thereafter average delay shoots up.

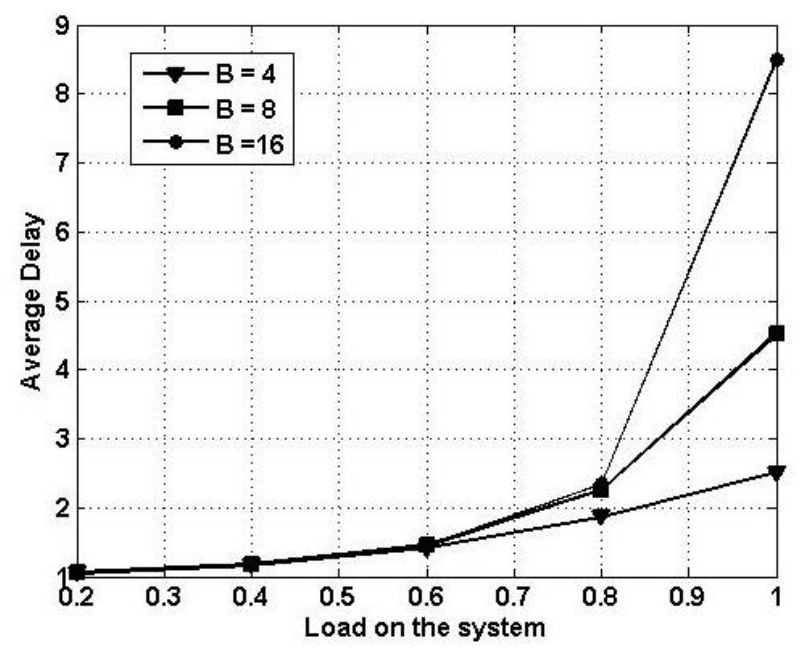

Fig.12. Average Delay vs. Load on the System with Varying Buffering Capacity

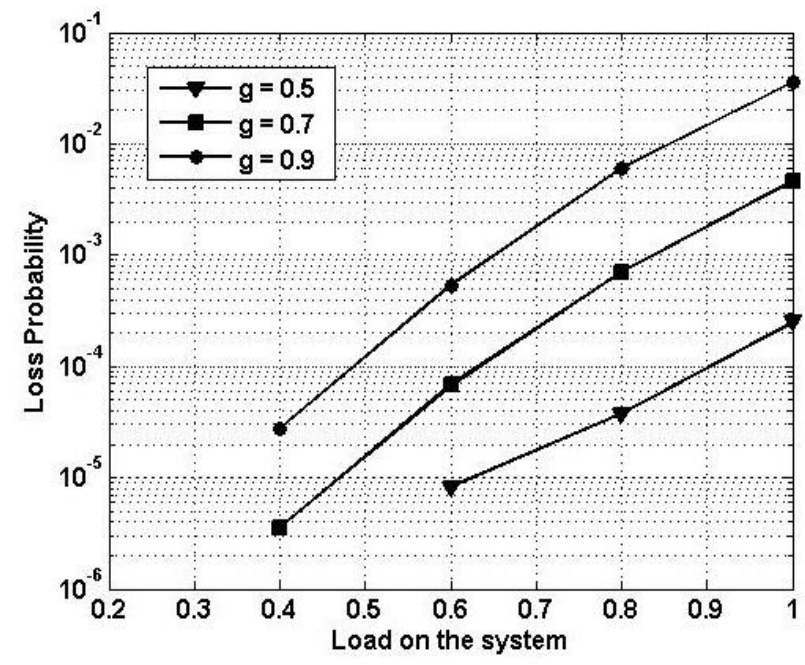

Fig.13. Packet Loss Probability vs. Load on the System with Fix Buffering Capacity of 4, with Load Balancing

In Fig. 13, packet loss probability vs. load on the system is presented while assuming $4 \times 4$ node i.e., 4 input and 4 output and buffering of 4 packets. However, load balancing scheme is applied. In the figure ' $g$ ' represents the fraction of traffic routed to global network. For global factor ' $g=0.5$ ' the packet loss probability, improves by a factor of 100 in comparison to ' $\mathrm{g}=0.9$ '.

In Fig. 14, average delay vs. load on the system is presented for similar configuration as in figure 13 . Comparing with figure 12 , the average delay has reduced considerably and this difference is more prominent at the higher loads. For ' $g=0.5$ ' the average delay at the load one is reduced by approximate 7 slots. Similarly results for ' $g=0.9$ ', where the average delay is recued by 6 slots. It must be remembered that, load balancing scheme reduces loss probability and average delay on local node only, however propagation delay increases as some part of the traffic will follow different path.

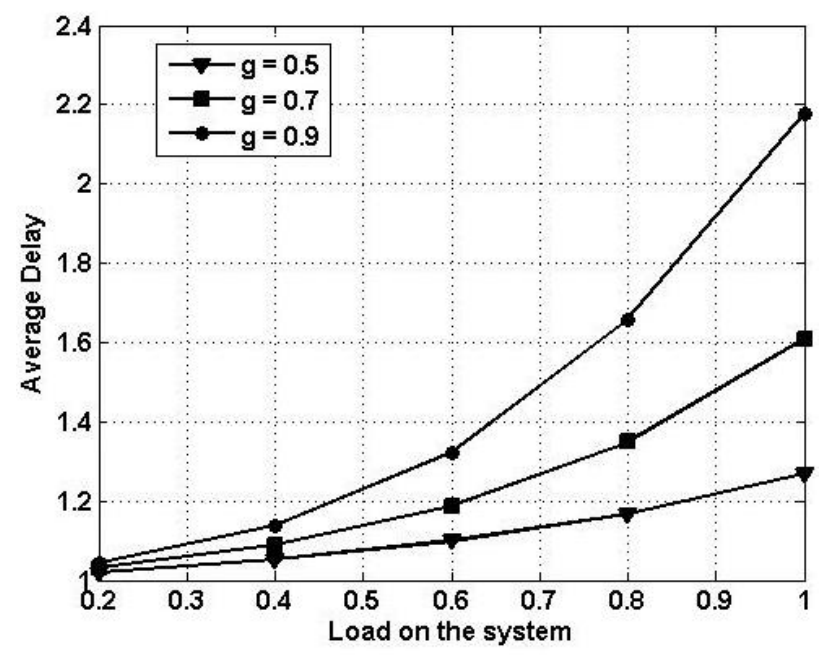

Fig.14. Average Delay vs. Load on the System with Fix Buffering Capacity of 4, with Load Balancing

\section{B. Shared Buffering}

In figure 15-18, results for shared buffering scheme are presented. In figure 15, packet loss probability vs. load on the system is presented while assuming $4 \times 4$ node i.e., 4 input and 4 output and buffering of 4,8 and 16 packets. At the load of 0.8 , with buffering capacity of 4,8 and 16 packets, the packet loss probability is $\square 4 \times 10^{-2}, \square 9 \times 10^{-3}$ and $\square 1 \times 10^{-4}$ respectively.

Thus by increasing the buffer space by four fold i.e., from 4 to 16 , the packet loss probability is improved by a factor of 400. In case of the output buffering at the load of 0.8 , the packet loss rate is $3 \times 10^{-5}$, however in case of shared buffering the packet loss rate is $\square 1 \times 10^{-4}$. Therefore in case of output buffered system, packet loss better by a factor of three only. But in case of shared buffering the total buffer space is 16 , but in case of output queued system is 64 . Hence, the performance of the shared buffering is much superior than that of output queued system with much complex controlling algorithm.

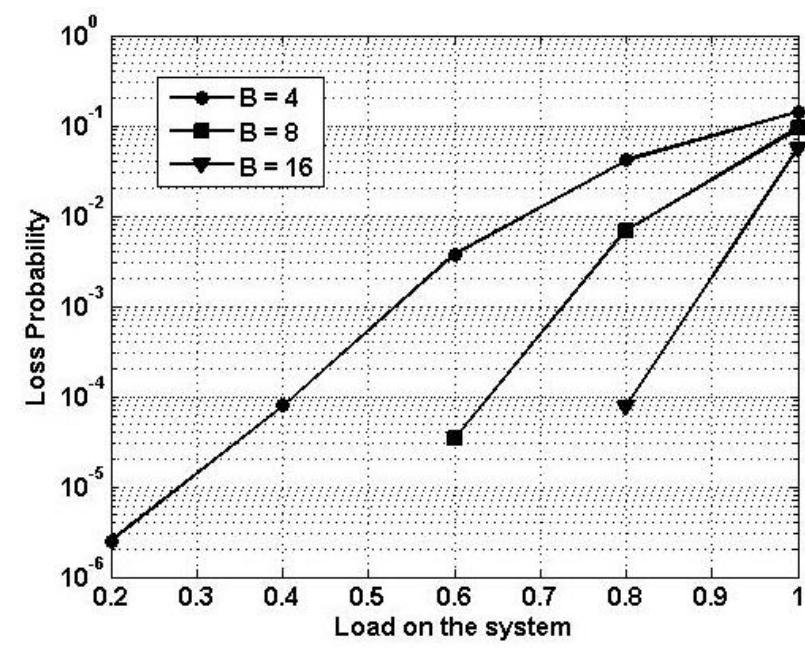

Fig.15. Packet Loss Probability vs. Load on the System with Varying Shared Buffering Capacity 


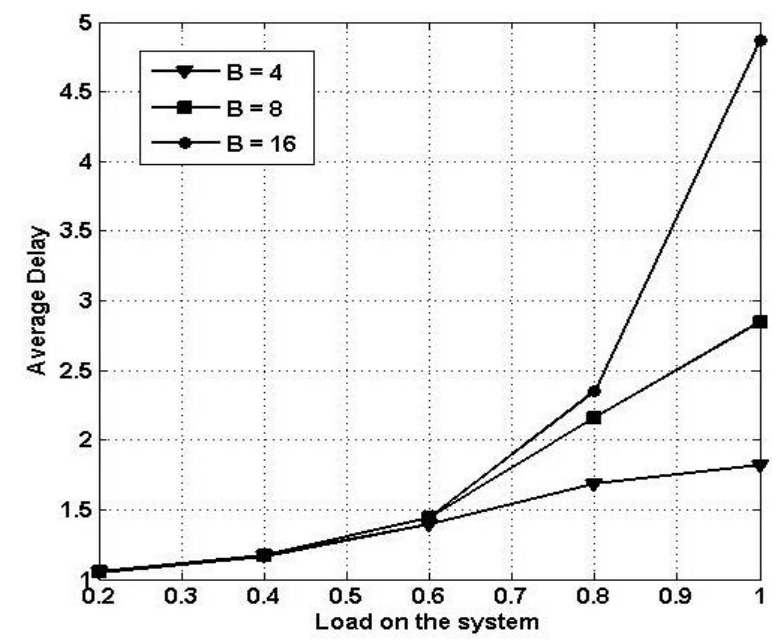

Fig.16. Average Delay vs. Load on the System with Varying Shared Buffering Capacity

In Fig. 16, average delay vs. load on the system is presented while assuming $4 \times 4$ node i.e., 4 input and 4 output and buffering of 4,8 and 16 packets. The average delay remains nearly same till load reaches 0.6 and in fact average delay is independent of buffering capacity. The average delay is of 5 slots for $B=16$, which is much less that the output queued system ( 8.5 slots). Therefore, the average delay performance of the shared buffer is much better than that of output queued system.

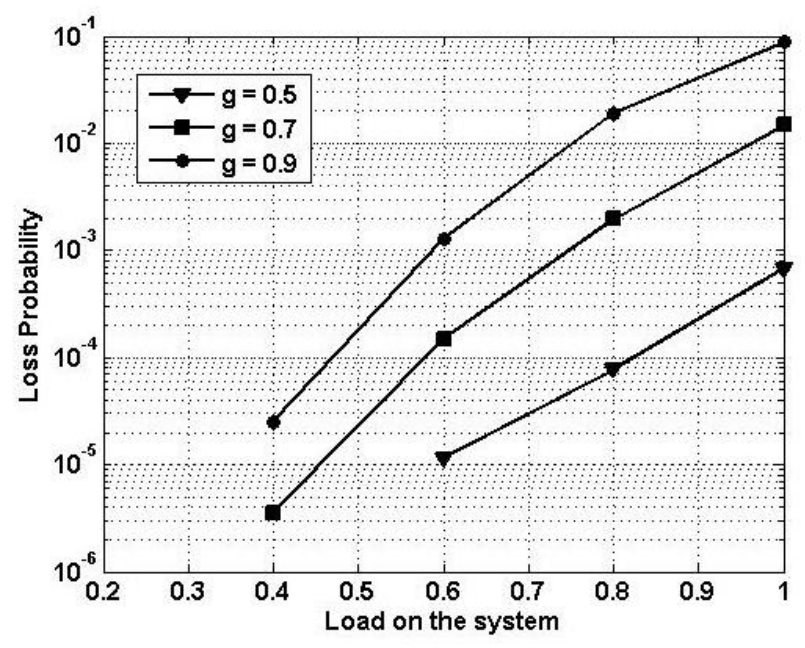

Fig.17. Packet Loss Probability vs. Load on the System with Fix Shared Buffering Capacity of 4, with Load Balancing

In Fig. 17, packet loss probability vs. load on the system is presented while assuming $4 \times 4$ node i.e., 4 input and 4 output and buffering of 4 packets in shared manner. In the figure ' $g$ ' represents the fraction of traffic routed to global network. At the load of 0.6, the packet loss performance of the shared and output buffered system is nearly same (Figure 13 and 17). Thus with much reduced buffering requirements shared buffering scheme, along with load balancing scheme provide very effective solution.

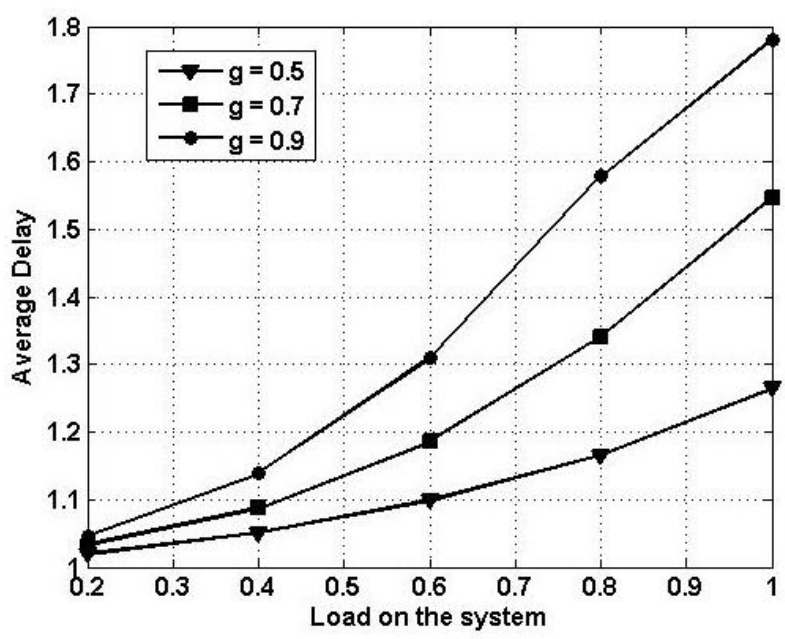

Fig.18. Average Delay vs. Load on the System with Fix Shared Buffering Capacity of 4, with Load Balancing

In Fig. 18, average delay vs. load on the system is presented while assuming $4 \times 4$ node i.e., 4 input and 4 output and shared buffering of 4 packets. However, load balancing scheme is applied. Comparing with figure 16 and 18 the average delay has reduced considerably and this difference is more prominent at the higher loads.

\section{CONCLUSIONS}

In this paper, an important switching problem, contention among the packet is discussed and possible solution: deflection routing and buffering of packets is detailed. For example a six node network is considered with 11 links. Simulation is performed to obtain packet loss rate and average delay and following conclusions can be made:

1. Buffering reduces the packet loss rate.

2. Average delay during buffering is much less then the propagation delay in the network.

3. Load balancing scheme reduces the loss rate at the contending node.

4. Deflection routing is needed when load balancing scheme is to be used to route packets to alternative paths.

5. Load balancing of data traffic reduces traffic at the contending nodes thus both loss rate and average delay reduces.

6. However, due to the load balancing need of deflection routing may enhance as more numbers of packets will follow different paths.

Thus it can be concluded that buffering is an essential and important part in optical packet switching systems.

\section{REFERENCES}

[1] Renaud, F. Masetti, C. Guillemot, and B. Bostica, "Network and System Concepts for Optical Packet Switching," IEEE Communications Magazine, vol. 35(4), pp. 96-102, April 1997.

[2] D.K. Hunter and I. Andonovic, "Approaches to Optical 
Internet Packet Switching," IEEE Communications Magazine, vol. 38(9), pp. 116-122, September 2000.

[3] S. Yao, B. Mukherjee, and S. Dixit, "Advances in Photonic Packet Switching: an Overview," IEEE Communications Magazine, vol. 38(2), pp. 84-94, February 2000.

[4] R. K. Singh, R. Srivastava and Y. N. Singh, "Wavelength division multiplexed loop buffer memory based optical packet switch," Optical and Quantum Electronics, vol. 39, no. 1 pp. 15-34, Jan. 2007.

[5] Rajiv Srivastava and Yatindra Nath Singh, "Feedback Fiber Delay Lines and AWG Based Optical Packet Switch Architecture," Journal of Optical Switching and Networking, volume 7, Issue 2, Pages 75-84, April 2010.

[6] Pallavi S , Dr. M Lakshmi: "AWG Based Optical Packet Switch Architecture "I.J. Information Technology and Computer Science, 2013, 04, 30-39 Published Online March 2013 in MECS (http://www.mecs-press.org/) DOI: 10.5815/ijitcs.2013.04.04

[7] M. G. Hluchyj, and M. J. Karol, "Queuing in high performance packet switching," IEEE J. in Sel. Areas Commun. vol. 6, no.9, pp. 1587-1597, Sep. 1988.

[8] Rajiv Srivastava, Rajat Kumar Singh and Yatindra Nath Singh, "WDM based optical packet switch architectures," Journal of Optical Networking, Vol. 7, No. 1, pp. 94-105, 2008.

[9] Rajiv Srivastava, Rajat Kumar Singh and Yatindra Nath Singh, "Optical loop memory for photonic switching application;" Journal of Optical Networking, Vol. 6, No. 4, pp. 341-348, 2007.

[10] Rajiv Srivastava, Rajat Kumar Singh and Yatindra Nath Singh, "Fiber optic switch based on fiber Bragg gratings" IEEE Photonic Technolgy Letters, Vol. 20, No. 18, pp. 1581-1583, 2008.

[11] Jun Huang, Yanwei Zhang, Xiaojin Guo "QoS Analyze on a Novel OTDM-WDM Optical Packet Switching" Journal of Computational Information Systems Vol., No.4 pp.1380-1386,2011.

[12] Vaibhav Shukla ; A. Jain ; R. Srivastava, "Physical layer analysis of an Arrayed Waveguide Gratings based Optical Switch", International Journal of Applied Engineering and Research, RIP Publisher, Vol. 9, No. 21, pp. 10035-10050, 2014.

[13] Houman Rastegarfar, Alberto Leon-Garcia, Sophie LaRochelle, and Leslie Ann Rusch "Cross-Layer Performance Analysis of Recirculation Buffers for Optical Data Centers,"Journal of Lightwave Technology, Vol. 31, Issue 3, pp. 432-445, 2013.

[14] G. Appenzeller, N. McKeown, J. Sommers, and P. Barford, "Recent results on sizing router buffers," in Proc. Network Systems Design Conf.,San Diego, CA, Oct. 1820, p. 13, 2004

[15] D. K. Hunter, M. C. Chia, I. Andonovic, "Buffering in optical packet switches", IEEE/OSA Journal of Lightwave Technology, vol. 16, no. 12, pp. 2081-2094, Dec. 1998.

\section{Authors' Profiles}

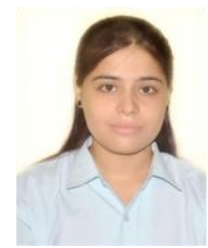

Chitra Sharma: Received her B.Tech in Information Technology from Krishna Girls Engineering College, 2013. She is studying M.Tech in Computer Science and Engineering from Kanpur Institute OF Technology. Her research interests are in the areas of computer networks, software engineering.

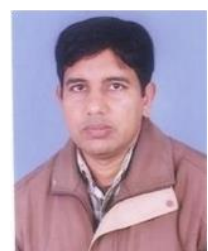

Akhilesh Kumar Yadav: Received his M.Tech in Computer science and engineering from MNNIT Allahabad, in 2004. He is assistant professor in the department of Computer science and engineering in the Kanpur Institute of Technology. His research interests are in the areas of computer networks, software engineering, software testing.

How to cite this paper: Chitra Sharma, Akhilesh Kumar Yadav,"Buffering Strategies in Optical Packet Switching", International Journal of Computer Network and Information Security(IJCNIS), Vol.8, No.3, pp.19-26, 2016.DOI: 10.5815/ijcnis.2016.03.03 